\title{
Plain X-Rays in The Diagnosis of Sickle Cell Limb Pain in Children
}

\section{A 10-Year Retrospective Audit}

\section{ABSTRACT}

Background and aims: Children with sickle cell disease (SCD) frequently present with limb pain. Differentials include vasoocclusive episode (VOE) and osteomyelitis (OM). X-rays expose to radiation, but rarely aid in diagnosis. We audited the use of $x$-ray in investigating children with SCD presenting with limb pain to a South London hospital, and analysed whether x-rays aid in diagnosis.

Methods: Patients aged 0-18 years with SCD were identified using the hospital's SCD database. Admissions from January 2010 to September 2019 in which limb pain was a documented symptom were included.

Results: Of 342 patients investigated, there were 188 admissions with limb pain. Diagnoses at discharge were: $174 \mathrm{VOE}, 4 \mathrm{OM}$, and 7 others. 44 (25\%) of those with VOE had limb x-rays, compared with $3(75 \%)$ of those with OM. Of those $x$-rayed, 11 with VOE and all with $\mathrm{OM}$ had a subsequent MRI. None of the $\mathrm{x}$-rays assisted in confirming diagnosis or changed management. Of the VOE patients, limb swelling ( $48 \%$ vs $8 \%, p=<0.0001)$ ), fever ( $57 \%$ vs $37 \%, p=0.021)$, and peak C -reactive protein (CRP) (109 vs $75 \mathrm{~g} / \mathrm{L}, \mathrm{p}=0.044$ ) were determinants of having plan $\mathrm{x}$-rays on admission.

Conclusions: X-rays were frequently used to investigate children with SCD but did not appear to influence diagnosis of OM or management. Those with suspected OM should undergo MRIs rather than plain $\mathrm{x}$-rays and be included in new clinical algorithms.

Keywords: sickle cell disease, vaso-occlusive crisis, osteomyelitis, Xray
Frederik Vivian, Subarna

Chakravorty*

Department of Paediatric Haematology,

King's College Hospital NHS Trust

*Corresponding author subarna.chakravorty@nhs.net

Cite as: Vivian, F., \& Chakravorty, S. (2021) Plain $\mathrm{x}$-rays in the diagnosis of sickle cell disease limb pain in children. A 10-year retrospective audit. The Physician vol 7; Issue 1: 1-6 ePub 27.02.2021 DOI: https://doi.org/10.38192/1.7.1.4
Article Information

Submitted 26.02.2021

ePub 27.02.2021 


\section{Introduction}

Sickle cell disease (SCD) is an autosomal recessive blood disorder characterised by frequent episodic pain, increased susceptibility to infection and chronic organ damage. Prevalence in the UK is approximately 1 in 4600, most commonly affecting people of African or AfricanCaribbean origin ${ }^{1}$. Painful vaso-occlusive episodes (VOE) are the most common cause for admission to hospital ${ }^{2}$. Recurrent VOE can result in bone infarction and osteonecrosis. People with $\mathrm{SCD}$ are also at increased risk of osteomyelitis (OM) for reasons including asplenia causing increased susceptibility to encapsulated bacteria, presence of infarcted bone due to osteonecrosis and potential hematogenous spread of gut pathogens due to ischaemia in the gut associated with vaso-occlusion ${ }^{3,4}$.

It is often difficult to distinguish VOE and OM clinically. Both present with musculoskeletal pain; and swelling, fevers and raised inflammatory markers are common features in both conditions. For a definitive diagnosis of $\mathrm{OM}$, positive blood or operative cultures (such as bone biopsy or joint aspirate) are required. However, blood cultures are often negative despite $\mathrm{OM}$, and bone biopsy is invasive and therefore cannot be used routinely. Radiological evidence can support a diagnosis of OM but plain film x-rays of the limb have a poor sensitivity and specificity for these ${ }^{5}$. Magnetic resonance imaging (MRI) or ultrasound are more likely to guide management, although these can also cause diagnostic uncertainty ${ }^{6}$.

In addition to poor diagnostic yield, plain film $\mathrm{x}$-rays of limbs result in exposure to ionising radiation and is particularly significant in children, who have greater sensitivity to ionising radiation ${ }^{7}$. In patients with SCD, where many presentations with musculoskeletal pain are likely, there is a significant risk from the cumulative radiation dose. Plain $\mathrm{x}$-rays are of little diagnostic benefit in distinguishing $V O E$ from $O M$ and yet they are frequently used as a first line investigation to exclude $\mathrm{OM}$ in SCD children presenting with limb pain. An exploration of the effectiveness of plain $x$-rays in the investigation of limb pain would help future clinical practice.

\section{Method}

We undertook an audit of the effectiveness of plain film $x$-ray to diagnose OM in children with SCD admitted with limb pain to a South London teaching hospital. The retrospective audit covered a period of nearly 10 years between January 2010 and September 2019. Patients with homozygous sickle cell disease and aged 0 to 18 years were identified from the paediatric sickle cell database and were included in the study. The study was approved by our Institutional review board as an audit.

For each admission, data on clinical features, final diagnoses (OM or VOE) and laboratory and radiology results were identified from the hospital electronic records. Results were analysed by final diagnosis and presence/absence of $\mathrm{x}$-ray use.

Statistical significance of differences in clinical features with binomial frequencies (presence of swelling and presence of fever) were calculated using a Chi-squared test. Significance of differences in normally distributed laboratory results was calculated using an unpaired ttest.

\section{Results}

A total of 342 patients on our clinical database were analysed with 584 admissions. Of these, 186 admissions had limb pain as a documented symptom, and were included in the audit. Of the 186 admissions for limb pain, the diagnoses at discharge were: 174 vasoocclusive episodes (VOE), four osteomyelitis (OM), three septic arthritis (SA), two avascular necrosis of the hip, one each of transient synovitis, myositis and non-specific wrist pain.

Imaging

Plain film $x$-rays were used in 56 of the 186 admissions (30\%), with 18 (9.7\%) also having MRI scans. Forty-four of the patients admitted for VOE (25\%) had x-rays [Table 1]. Seven patients with $V O E$, all patients with $O M$ and $S A$, went on to having MRIs. None of the x-rays appeared to have contributed to the final diagnosis or changed management.

Vaso-occlusive episodes 
Patients presenting with limb pain and a diagnosis of VOE were more likely to be investigated with limb x-rays if they had swelling of the affected limb (48\% vs $8 \%$, $p=<0.001)$, fever ( $57 \%$ vs $37 \%, p=0.021)$ and an elevated mean C-reactive protein level (109 vs $75 \mathrm{~g} / \mathrm{L}, \mathrm{p}=0.044$ ), with no significant difference in total white cell count.

\section{Osteomyelitis}

All patients with OM presented with localised swelling and three out of four presented with fever. Peripheral blood cultures were sent in $45 \%$ of patients with VOE, all patients with OM and SA. Of the $45 \%$ of VOE patients where blood culture were taken, 3 had positive results growing organisms that are frequent contaminants [Table 2]. Only one of the patients with OM had a positive peripheral blood culture result while another was positive from a bone biopsy.

\section{Pain}

All patients with OM had unilateral limb pain, compared with VOE patients where the median was pain affecting more than 2 sites. The number of painful sites was not found to be a determinant of who had x-rays. Patients with OM had a longer duration of pain prior to admission, compared to those with VOE (8 vs 2.47 days) [Table 3].

\section{Antibiotics}

In this audit, 97 children (56\%) with a diagnosis of VOE received broad spectrum antibiotics for a mean duration of 7 days, with some of these having alternative indications for antibiotic treatment.

\section{Discussion}

While OM is a serious diagnosis in children with $S C D$, it is rare compared to vaso-occlusive pain mimicking $\mathrm{OM}^{4}$. $\mathrm{X}$ rays do not appear to aid in this diagnostic challenge, due to low sensitivity for both conditions and lower specificity. MRI scans can be helpful, and are an important investigation in OM, although infarcted and infected bone can appear similar in this modality ${ }^{3}$. Positive blood cultures with pathogenic bacteria improves confidence of a diagnosis of OM, but the sensitivity of blood cultures is relatively low. Culture of an operative biopsy sample is a definitive investigation to confirm OM, but due to its invasive nature, is not appropriate unless there is a very high clinical suspicion 6 . The investigation of children with bone pain and infective symptoms is therefore a difficult area for paediatricians and haematologists.

In our analysis of admissions for limb pain, 25\% of patients with VOE had a plain x-ray. Plain x-rays offered no benefit to the diagnostic process. Those patients presenting with features suggestive of OM such as an elevated CRP, fever or limb swelling were likely to have a plain x-ray as their initial investigation. In none of the 186 admissions, did a limb x-ray aid diagnosis or alter management. Thus in such cases of suspected OM, an MRI of the affected limb would be a more appropriate investigation.

Children with suspected OM are treated with broad spectrum empirical antibiotics, and many of these are discontinued once clinical suspicion of OM reduces during the admission episode. There appears to be variation in institutional practices in the use of empirical antibiotics in SCD children presenting with limb pain. In this audit, $56 \%$ of children received broad spectrum antibiotics compared to $86 \%$ of 358 VOE patients in another paediatric cohort from London ${ }^{8}$.

In this audit we examined admissions of patients on our current SCD paediatric database over a 10-year period. This therefore excluded admissions of patients that occurred within the last 10 years, but that are now older than 18 and are therefore no longer in our current database. This resulted in an age-skew of admissions, with more admissions of younger patients. While VOE commonly presents from a young age onwards, OM is more likely in older children. Since the purpose of the audit was to examine the plain $x$-ray use in children presenting with limb pain, we chose to study all children with pain, rather than identify those with proven or probable OM and work retrospectively to their original presentation. A longer study looking at the clinical features and investigation variation between VOE and OM may offer greater insight into how these can be distinguished and is an area that would benefit from further research. 
We have found a high frequency of x-ray (30\%) use for investigation of limb pain in children with sickle cell disease resulting perhaps in unnecessary irradiation in a population at risk of significant lifetime exposure. Evidence based clinical algorithms or guidelines for suspected OM incorporating features such as fever, unilateral / single site of pain and raised inflammatory markers may help target appropriate imaging such as $M R I$ and reduce the use of plain $x$-rays in the management of limb pain in children with SCD is needed. We plan to re-audit the use of plain x-rays once these measures are put in place and hope to achieve a reduction in $\mathrm{x}$-ray use in this group of children.

\section{$\underline{\text { References }}$}

1 Dormandy, E., James, J., Inusa, B. \& Rees, D. How many people have sickle cell disease in the UK? J Public Health (Oxf) 40, e291-e295, doi:10.1093/pubmed/fdx172 (2018).

2 Platt, O. S. et al. Pain in sickle cell disease. Rates and risk factors. N Engl J Med 325, 11-16, doi:10.1056/NEJM199107043250103 (1991).

3 Kosaraju, V. et al. Imaging of musculoskeletal manifestations in sickle cell disease patients.
Br J Radio/ 90, 20160130, doi:10.1259/bjr.20160130 (2017).

4 Burnett, M. W., Bass, J. W. \& Cook, B. A. Etiology of osteomyelitis complicating sickle cell disease. Pediatrics 101, 296-297 (1998).

5 Al Farii, H., Zhou, S. \& Albers, A. Management of Osteomyelitis in Sickle Cell Disease: Review Article. J Am Acad Orthop Surg Glob Res Rev 4, e2000002-2000010, doi:10.5435/JAAOSGlobalD-20-00002 (2020).

6 Vanderhave, K. L., Perkins, C. A., Scannell, B. \& Brighton, B. K. Orthopaedic Manifestations of Sickle Cell Disease. J Am Acad Orthop Surg 26, 94-101, doi:10.5435/JAAOS-D-16-00255

(2018).

$7 \quad$ Kleinerman, R. A. Cancer risks following diagnostic and therapeutic radiation exposure in children. Pediatr Radiol 36 Suppl 2, 121-125, doi:10.1007/s00247-006-0191-5 (2006).

8 Fontalis, A. et al. The challenge of differentiating vaso-occlusive crises from osteomyelitis in children with sickle cell disease and bone pain: A 15-year retrospective review. J Child Orthop 13, 33-39, doi:10.1302/1863-2548.12.180094 (2019). 
Table 1: Frequency and percentage of plain film $x$-ray use, by final diagnosis

\begin{tabular}{|l|l|l|}
\hline Final diagnosis at discharge & Number & Frequency of $\mathbf{x}$-ray use (percentage) \\
\hline VOE & 174 & $44(25)$ \\
\hline OM & 4 & $3(75)$ \\
\hline Septic arthritis & 3 & $2(67)$ \\
\hline Myositis & 1 & $1(100)$ \\
\hline AVN & 2 & $2(100)$ \\
\hline Transient synovitis & 1 & $1(100)$ \\
\hline Wrist pain & 1 & $0(0)$ \\
\hline Total & 186 & $56(30)$ \\
\hline
\end{tabular}


Table 2: Organisms isolated from blood culture, by final diagnosis

\begin{tabular}{|l|l|}
\hline Diagnosis & Organisms isolated (from blood cultures unless stated) \\
\hline VOE & Staphylococcus caprae, Staphylococcus epidermidis, Streptococcus viridans \\
\hline OM & Abiotrophia defectiva, Salmonella (negative blood cultures, positive intra-operative sample) \\
\hline
\end{tabular}

Table 3: Clinical features and investigations, by final diagnosis

\begin{tabular}{|c|c|c|c|c|c|c|}
\hline & $\begin{array}{l}\text { VOE } \\
\text { (all) }\end{array}$ & $\begin{array}{l}\text { VOE } \\
\text { ( } \geq 1 \text { limb x-ray) }\end{array}$ & \begin{tabular}{|l} 
VOE \\
(no limb x- \\
ray)
\end{tabular} & OM & SA & Other \\
\hline Number & 174 & 44 & 130 & 4 & 3 & 5 \\
\hline Number (\%) females & 57 (33) & $13(30)$ & $44(34)$ & $3(75)$ & $1(33)$ & $2(40)$ \\
\hline $\begin{array}{l}\text { Number }(\%) \text { on } \\
\text { hydroxycarbamide }\end{array}$ & $93(53)$ & $27(61)$ & $66(51)$ & $2(50)$ & $1(33)$ & $2(40)$ \\
\hline Number (\%) on penicillin & $162(93)$ & 41 (93) & $121(93)$ & $4(100)$ & $3(100)$ & $5(100)$ \\
\hline $\begin{array}{l}\text { Mean age at admission, years } \\
\text { (s.d.) }\end{array}$ & $8.26(4.7)$ & $7.45(5.6)$ & $8.54(4.4)$ & $14.5(1.7)$ & $7.33(3.5)$ & $5.6(5.5)$ \\
\hline Limb x-ray used & $25 \%$ & $100 \%$ & $0 \%$ & $75 \%$ & $66.7 \%$ & $80 \%$ \\
\hline MRI used & $7 \%$ & $25 \%$ & $0.8 \%$ & $75 \%$ & $66.7 \%$ & $40 \%$ \\
\hline Peak WCC, mean (s.d.) & $14.8(8.0)$ & $16.6(12.3)$ & $14.2(5.8)$ & $12.3(3.0)$ & $22.3(2.1)$ & $14.4(6.7)$ \\
\hline Peak CRP, mean (s.d.) & $84.3(95.3)$ & $\begin{array}{l}113.2(90) \\
p=0.044^{*}\end{array}$ & $74.5(95.5)$ & 77.5 (111.1) & $130.3(16.9)$ & $68.2(106.6)$ \\
\hline Swelling present & $18 \%$ & $48 \%(p<0.001)^{*}$ & $8 \%$ & $100 \%$ & $100 \%$ & $40 \%$ \\
\hline Fever present & $42 \%$ & $57 \%(p=0.021)^{*}$ & $37 \%$ & $75 \%$ & $67 \%$ & $0 \%$ \\
\hline Max. height of fever, ${ }^{\circ} \mathrm{C}$, mean & 38.4 & 38.3 & 38.5 & 38.5 & 38.4 & $\mathrm{n} / \mathrm{a}$ \\
\hline Blood Cultures sent & $45 \%$ & $62 \%$ & $39 \%$ & $100 \%$ & $100 \%$ & $20 \%$ \\
\hline Positive Blood Cultures & 3 & 1 & 2 & 1 & 0 & 0 \\
\hline \begin{tabular}{|l|} 
Biopsy / Aspiration \\
\end{tabular} & $0.57 \%$ & $2.27 \%$ & $0 \%$ & $50 \%$ & $50 \%$ & $0 \%$ \\
\hline $\begin{array}{l}\text { Mean number of sites affected } \\
\text { (s.d.) }\end{array}$ & $2.5(1.2)$ & $2.3(1.3)$ & $2.6(1.2)$ & $1(0)$ & $2.0(1)$ & $1.2(0.4)$ \\
\hline $\begin{array}{l}\text { Mean duration of pain prior to } \\
\text { admission, days (s.d.) }\end{array}$ & $2.5(3.2)$ & $3.2(4.6)$ & $2.2(2.5)$ & $8(9.0)$ & $3.3(3.2)$ & 19.2 (39.6) \\
\hline
\end{tabular}

$O M=$ osteomyelitis, $S A=$ septic arthritis, s.d.= standard deviation, $W C C=$ white cell count, $C R P=C$-reactive protein

*Patients with VOE who received x-rays were more likely to have higher mean CRP (113.2 vs 74.5, p=0.44), swelling of the affected limb (48\% vs $8 \%, p=<0.001)$ and fevers ( $57 \%$ vs $37 \%, p=0.021$ ) 